\title{
Development of an OPC and PLC Based Remote-Access Laboratory: A Synchronous Motor Control Experiment
}

\author{
S.Vadi $* 1$, R. Bayındır ${ }^{2}$ \\ Accepted $15^{\text {th }}$ August 2014 \\ DOI: 10.18100/ijamec.88432
}

Abstract: In this study, OPC and PLC based remote-access laboratory has been developed for synchronous motor control experiment. The monitoring and control of the parameters of synchronous motor has been realized using GPRS and Profi-Lab OPC Server through the visual programming language. In addition, S7-1200 CPU 1214 DC/DC/DC series PLC was used for control of the system. The motor parameters monitored in real-time by the user to visually is shown the effect of any change in the parameters of the motor with the help of the user visual interface. This laboratory carried out monitoring and control of a motor rotating motion and more safely by conventional methods. In addition, the experiments are realized GPRS communication method using a synchronous motor without a physical contact between the users. Thanks to the wireless access system, hardware complexity has been eliminated.

Keywords: Synchronous motor, OPC, GPRS, Remote access, PLC.

\section{Introduction}

Rapidly developing technology presents innovations in many areas such as electrical, electronic, software and power electronics. Classical methods and systems are replaced by more modern infrastructure. Especially, thanks to advanced computer programs and computer systems in industrial automation systems are easy technique with computer controlled or the remote control using industrial equipment [1-3]. The control processing are performed with wired communication systems such as USB, serial or parallel port etc. and also wireless communication systems such as GPRS, Bluetooth, infrared etc. [4-8]. The wired communication system with different products in large scale project causes hardware complexity. Therefore the wireless communication is more preferable methods than the wired communication [9].

Bluetooth, infrared, and wireless communication systems are used in small size data transfer capacity and in small projects due to its low coverage area [10]. GPRS can also be used areas where with an internet connection because of providing fast and uninterrupted connection. Therefore, in this study was used GPRS communication [11-14]. However, the complexity emerges in a variety of industrial products, and each product uses a separate driver or program. However, OPC (Ole for Process Control) foundation helps define the standard through working groups, and by creating and maintaining open specifications to standardize communication. The OPC (Open Platform Communications) is a standard for industrial communications that enables universal connectivity and interoperability. In addition, the OPC technology is based on Client/Server architecture and provides standards-based communication that allows users to employ a "best of breed" solution for their

\footnotetext{
${ }^{I}$ Depertmant of Electrical and Energy, Vocational College of Gazi, Gazi University, Çubuk, Ankara, Turkey.

* Corresponding Author: Email: seyfettinvadi@gazi.edu.tr

\# This paper has been presented at the International Conference on

Advanced Technology\&Sciences (ICAT'14) held in Antalya (Turkey),
}

projects. In essence, OPC is the Modbus of the new century [15, $16]$.

Many professions use OPC to help manage their processes. OPC is currently used in industries such as: power, oil \& gas, petrochemical, pulp \& paper, building automation \& HVAC, automotive manufacturing, pharmaceutical, military applications, and more. Therefore, in this study, the OPC standard was preferred for industrial communications of different manufacturers to communicate with each other to provide a common platform between the user interface and GPRS module because of providing an easy and flexible way communication. OPC, GPRS module communicates wirelessly used for data transfer to the user interface. In this way, industrial applications have reached systematic and reliable structure than conventional systems. In addition, the OPC provides at the least cost development and deployment in the development of existing industrial systems or the establishment of new systems due to universal structure and support of technological developments [17]. Thanks to, ability of use the visual platforms functions such as Matlab, Labview, Profilab and also programming languages functions Visual Basic, Visual C \#, Java, Delphi work integrated these programs [18-22]. A programming language need code writing for programming. Therefore, computer software engineers are generally use visual programming languages.

Microcontroller, FPGA, DAQ and PLC can be used in the remote-controlled systems as microprocessor in the literature [22]. Microcontroller-controlled systems are affected by adverse environmental conditions such as electromagnetic pollution, ambient noise. Therefore, microcontrollers are generally used in very simple and small projects [23]. In addition, the existing structure does not allow for the development of the ability of the system. A field-programmable gate array (FPGA) is an integrated circuit designed to be configured by a customer or a designer after manufacturing - hence "field-programmable".

FPGAs can be used to implement any logical function that an application-specific integrated circuit could perform. FPGA are used high-speed application and offer advantages for many 
applications [24].

Data acquisition (DAQ) is the process of sampling signals that measure real world physical conditions and converting the resulting samples into digital numeric values that can be manipulated by a computer. Data acquisition systems typically convert analog waveforms into digital values for processing. The components of data acquisition systems include sensors, signal conditioning circuitry to convert sensor signals into a form, and analog-to-digital converters. Regardless of the type of physical property to be measured, the physical state that is to be measured must first be transformed into a unified form that can be sampled by a data acquisition system [25]. The FPGA is only used for high-speed data acquisition and transfer operations, due to no internal memory. A programmable logic controller (PLC) or programmable controller is a digital computer used for automation of electromechanical processes, such as control of machinery on factory assembly lines, smart home applications, motor control applications and remote controlled applications. Unlike general-purpose computers, the PLC is designed for multiple inputs and output arrangements, extended temperature ranges, immunity to electrical noise, and resistance to vibration and impact [26].

In this study, remote control of synchronous motor has been realized based on OPC and PLC. GPRS communication method has been used for remote communication because of providing an easy and flexible way communication. GPRS module communicates wirelessly used for data transfer to the user interface. The most important difference of this study from literature, a wireless remote control and monitoring of the synchronous motor parameters has been provided. In this purpose, PLC has been used as controller due to flexible hardware and stability of working. The monitoring and control of the parameters of synchronous motor has been realized using GPRS and Profi-Lab OPC Server through the visual programming language. The motor parameters monitored in realtime by the user to visually are shown the effect of any change in the parameters of the motor with the help of the user visual interface.

\section{Hardware and Software Structure of System}

In this study, the remote access electrical machines laboratory was realized based on OPC and S7-1200 PLC. Block diagram of the overall system is shown in Fig.1. GPRS communication method is used for remote access. The Teleservice OPC Server program was used between the computer and the GPS module for communication. PLC was preferred monitoring and control of the system as controller unit due to its stable and fast operation. Parameters of motor are obtained by means of electronic cards and controlled by PLC controller and also are transferred to the OPC Server program using GPRS. All incoming measuring values defined in our study have been processed and displayed on the screen.

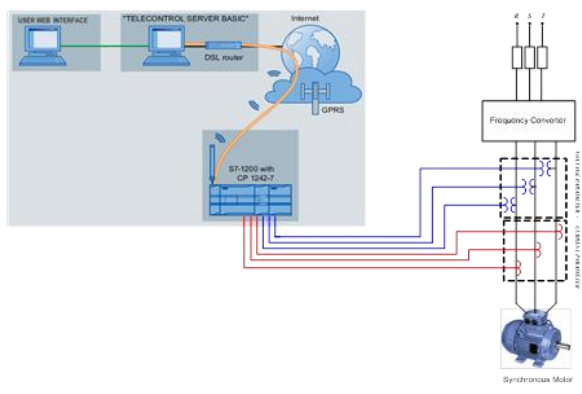

Figure.1 Block diagram of system

\subsection{Hardware Units}

\subsubsection{Controller Unit}

Programmable logic controller (PLC), microprocessor and microcontroller-based control systems are used in the control systems instead of electrical control circuits such as contactors, relays, and elements such as timers. PLC is preferred because of ease of use and programming. In addition, PLC control systems bring the solutions to complex control circuits, especially the PLC device is designed for industrial systems so on. Parts of the PLC hardware used in the industrial field are common. These parts are power supply, CPU (Central Processing Unit), input and output unit, program, data and communication interface.

In this study, Siemens S7-1200 1214C DC / DC / DC product series PLC is used for the processing and evaluating of data from the system. This PLC has 14 digital input/10 digital outputs, 2 analog inputs and has 8192 bytes of data memory. In addition, SM 1234 analog module series that works with compatible PLC is added due to not enough number of analog input channels. This module has 4 pieces analog inputs and 2 analog outputs. Analog channels have 13-bit resolution analog-to-digital conversion. The block diagram of the control unit is given Fig.2.

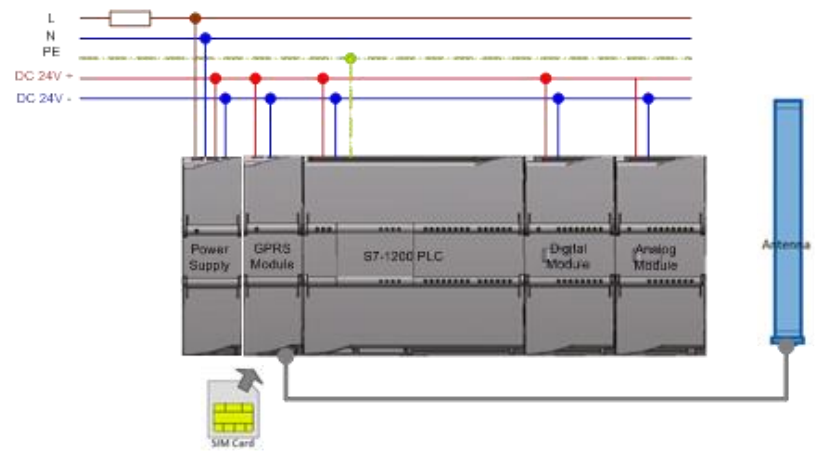

Figure 2. Overview of control unit

\subsubsection{GPRS Module}

The CP 1242-7 module adds a GSM/GPRS (Global System for Mobile Communications/General Packet Radio Service) networks interface to the Simatic S7-1200 controller. It enables plant operators to cost-effectively diagnose, maintain, monitor and control remote stations throughout the world via GPRS and Internet. The GPRS core network is the central part of the general packet radio service (GPRS) which allows $2 \mathrm{G}, 3 \mathrm{G}$ and WCDMA mobile networks to transmit IP packets to external networks such as the Internet. The GPRS system is an integrated part of the GSM network switching subsystem [27]. GPRS(General Packet Radio Service) is a technology that is provided high speed and from terminal to terminal data communication packet over the GSM grid. Data communication is possible from point to point or from point to multi-point. This technology is set up GSM substructure parallel and can access everywhere in GSM. GPRS technology is provided fast, easy, economic data communication. Because grid have a continuous connection to GPRS devices, Communication time is no. And, access data is easy so connection is ready not calling whatever number [28].

\subsubsection{Measurement Unit}

Electronic cards are designed to take synchronous motor parameters in controller platform. Current-Voltage Read Card is 
making to three phase current-voltage value. Also, to obtain power factor, zero-crossing circuit is done. Thanks to this dates, appear, active and reactive power of the synchronous motor is calculated. The block diagram of measurement card is given in Fig.3. Values of motor phase voltage are obtained with transformers. This transformer gives $220 / 5 \mathrm{~V}$ output voltage. Output voltage is rectified with bridge diode and this voltage is connected analogue input of controller. To obtain current values for every phase is used three numbers CASR-25NP current sensor. AC signals which is obtained from current and voltage card is connected zero-crossing card and power factor is calculated.

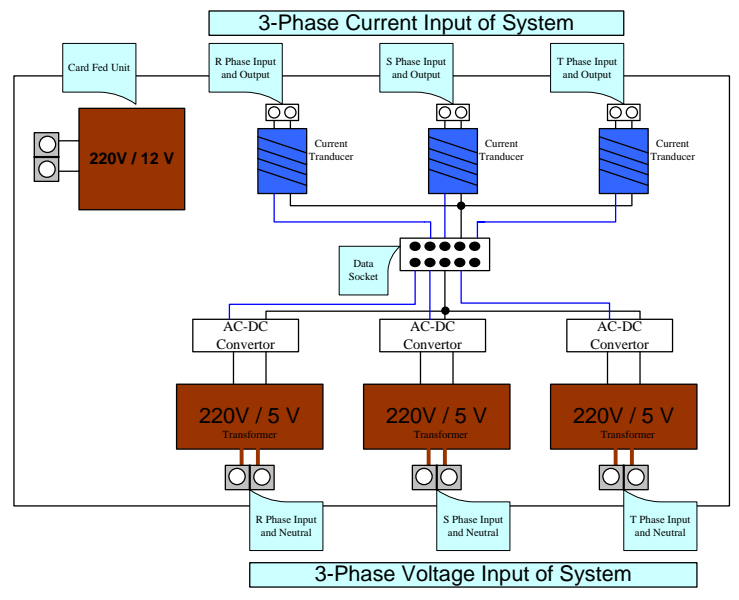

Figure 3. Block schema of the measurement card

Frequency convertor is used to provide development of the asynchronous motor, run different speeds and obtain speed and frequency of motor. Frequency converter is preferred because of it provides to concordance with PLC and responding to dynamic behavior as fast. Parameters of synchronous motor are given in Table-1. Together winding stator of synchronous motor apply to $\mathrm{AC}$, motor is run as asynchronous motor initially. To this process, frequency motor is used. After motor reached to nominal speed in asynchronous mode, friction winding of the synchronous motor is applying $0-110 \mathrm{~V}$ variable DC voltage. Thus, motor is provided to reach synchronous speed locking rotate field poles of stator and poles of rotor. All parameters that is obtained in run time are transfer to OPC Server over GPRS.

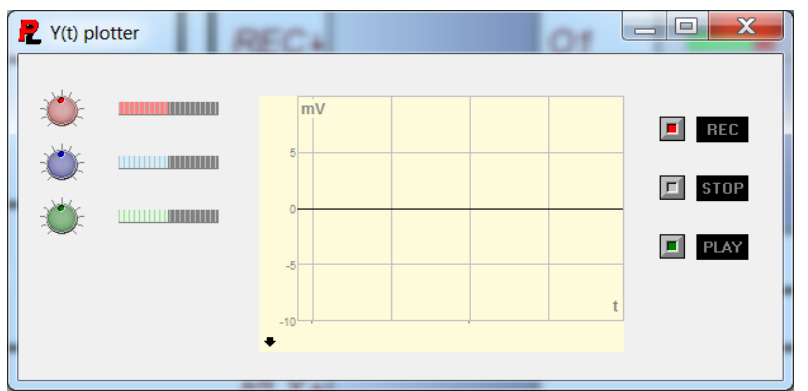

Figure 4. Profi-Lab Program screenshot and code page view
Table 1. Parameters of synchronous motor

\begin{tabular}{|c|c|}
\hline Nominal Power & $0,3 \mathrm{~kW}$ \\
\hline Nominal rpm & 1500 \\
\hline Power Factor & 0,97 \\
\hline Excitation Current & $0,95 \mathrm{~A}$ \\
\hline $\begin{array}{c}\text { Triangular Circuit } \\
\text { Connection Voltage / } \\
\text { Current }\end{array}$ & $400 \mathrm{~V} / 1,44 \mathrm{~A}$ \\
\hline
\end{tabular}

\subsection{Software Units}

\subsubsection{Profi-Lab Program}

Interface of the application is prepared in Profi-LAB platform which is graphical programmable language. This platform is help to development environment by code blocks. Thus, Very complex applications are possible to design basic level. Design and code blocks screenshot of the program is given Fig.4. As can be seen in the image, it is possible to create visual control and monitoring programs.

\subsubsection{Telecontrol Service Basic Program}

Telecontrol Service Basic Program is a product of Siemens firm. This program is used to provide a connection and transfer data to PC by Siemens CP1242-7 GPRS module. And it is run such as OPC Server. OPC is a standard name of communication interface in automation systems. A OLE sentence is mean a component of Microsoft and it has a structure using by functions of other platforms [29]. OPC is not a protocol. But, OPC is a standard approach based on data connectivity with its features. It refers to client. This standard features is supported by OPC Foundation. Together OPS Server is existing, all hardware and software manufactures haven't to share it internal structure of their systems by other firm and users. Thus, they can be opened by single standard interface the outside world. Another advantage of OPC put away limitations in selection of industrial devices and presents option freedom of the mark to end-user [30]. OPC is used wide-area such as sewage plants, drinking water distribution and purifies, the oil and gas stations, district heating systems, traffic control systems, air stations, wind turbine and building automation systems since it presents powerful and reliable platform to hardware and systems. Data transfer process is made downlink speed as 86kbit/s and uplink speed as $43 \mathrm{kbit} / \mathrm{s}$ in this communication interface as wireless $[29,30]$.

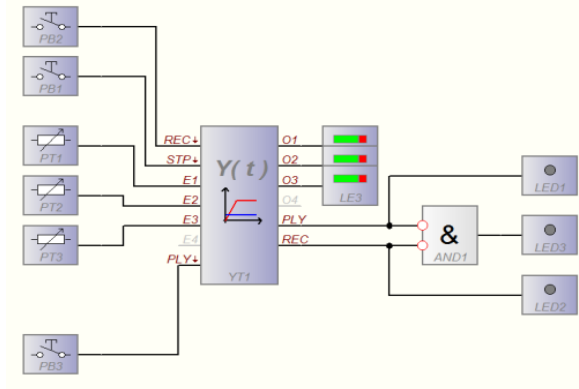




\section{Design And Application of the System}

\subsection{Design}

In this application is developed an OPC and PLC based remote- access laboratory for a synchronous motor control experiment. Block diagram belong to hardware structure of the system implemented is given Fig. 5.

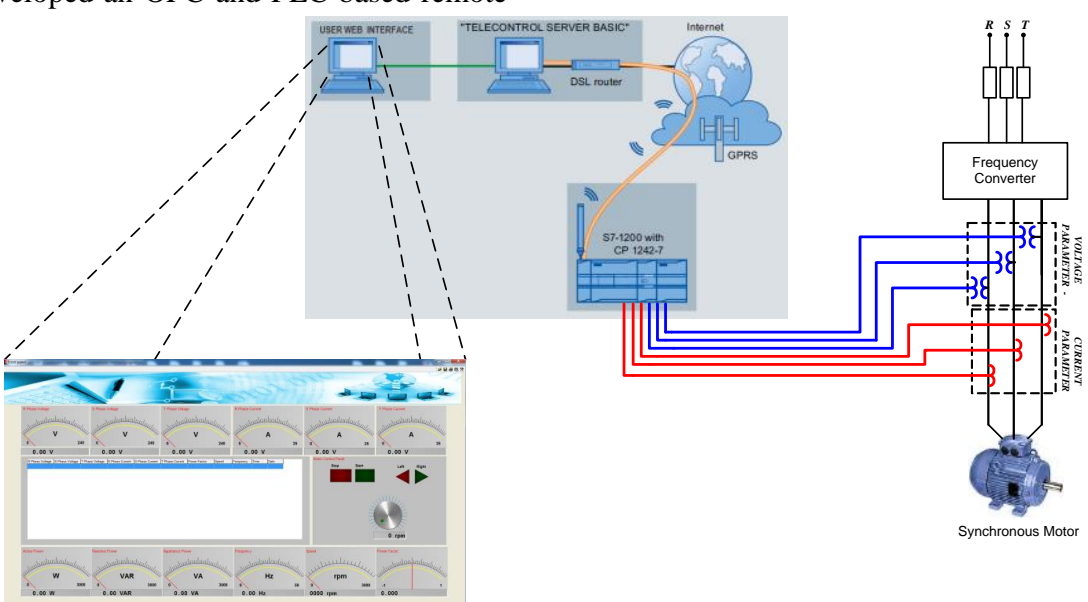

Figure 5. Hardware structure of the system implemented

\subsection{Implementation and Experimental Results}

In application implemented, to obtain parameters of synchronous motor is used electronic cards. After this data convert to digital form by SM 1234 analog module, it transfer to PLC. Thanks to Siemens CP1242-7 GPRS module which is

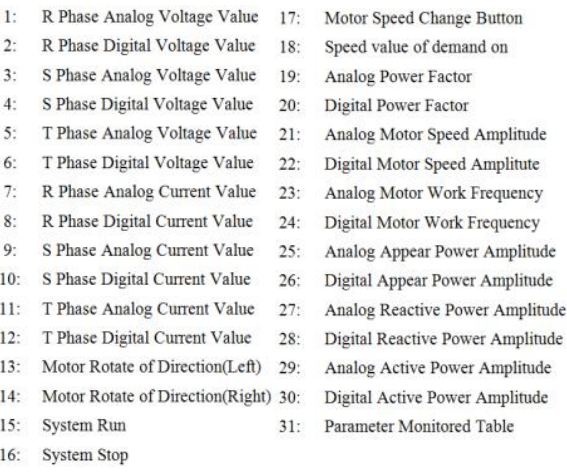

connected to PLC, data transfer to OPC Server. Users connect to OPC Server as client and data that is obtained is shown in screen visually. User interface and meaning of the motor parameters that is placed in user interface are given in Fig. 6.

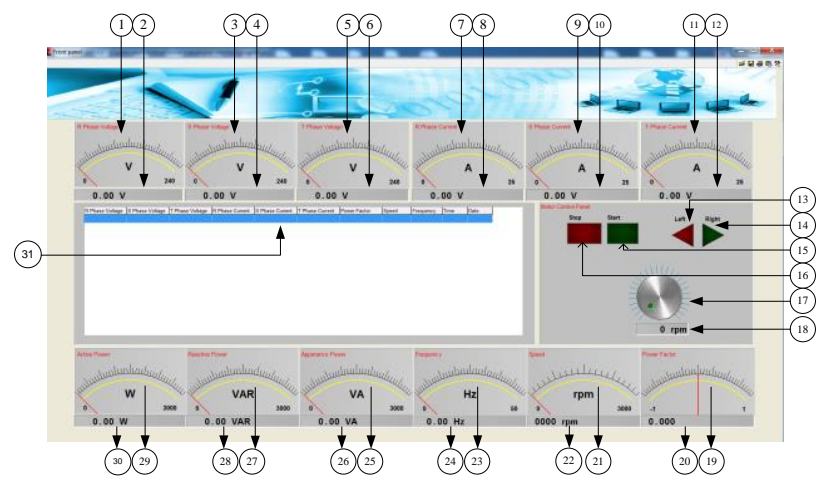

Figure 6. General View of the user interface and meaning of the motor parameters that is placed in user interface

While synchronous motor was nominal speed, user interface view is given Fig. 7. When user is clicked "start" button, user is connected to system automatically and is monitored three phase voltage and current values, active, reactive and appearance power values, running frequency and power factor values of the synchronous motor over interface.
Also, user is analyzed this parameters as digital and analogue. User is controlled start-stop process and process of change to direction of rotate and speed of motor as digitally. In addition, all parameters belong to motor is saved as a table in real time. When user is clicked "stop" button, motor is stop and connection is cut.

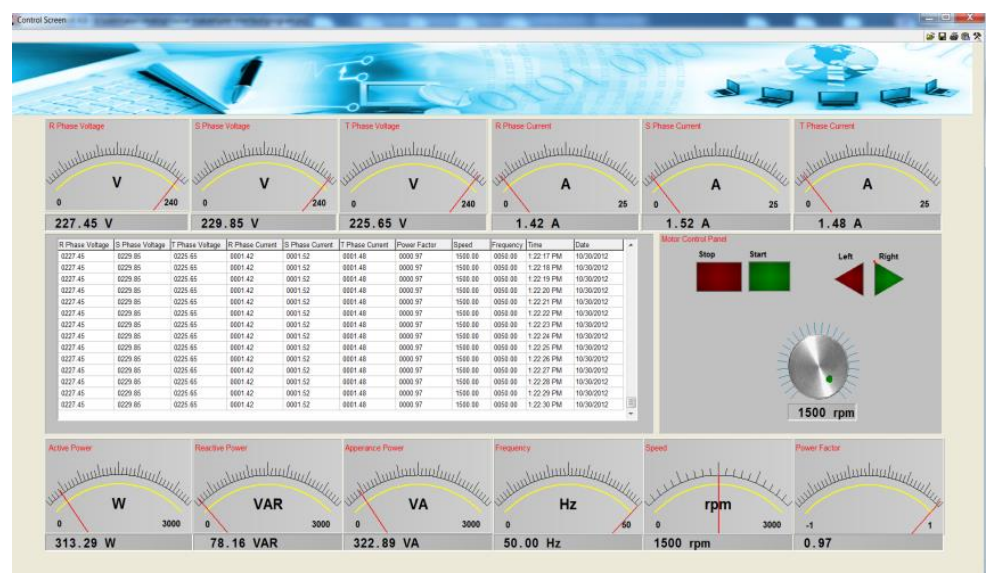

Figure 7. Screenshot of the synchronous motor that is rotated in nominal speed 


\section{Conclusion}

This study provides remote-access laboratory for synchronous motor control experiment base on OPC and PLC. The monitoring and control of the parameters of synchronous motor has been realized using GPRS and Profi-Lab OPC Server through the visual programming language. The experiments are realized GPRS communication method using a synchronous motor without a physical contact between the users.

Thanks to the wireless access system,

- Hardware complexity has been eliminated.

- The motor parameters has been monitored in real-time

- Using wireless communication more simple and more convenient environment has been provided.

- Parameter of motor has been observed through visual and graphical interface.

- Student has the opportunity to do the same experiment repeatedly.

- Time independent education environment has been obtained.

- It is achieved more effective and successful learning

In the future

- the application area of this work can be improved by adding additional hardware module.

- the application area of this work can be used to analyze motor in Research and Development Projects.

\section{References}

[1] Kurtkaya, O., Dağ, H., 1600 kW, 6300V Implemented of Synchronous Motor Excitation System with PLC, İstanbul Technic University- Institute of Science, Master Thesis, 2006.

[2] Ioannides M. G., Design and Implementation of PLC-Based Monitoring Control System for Induction Motor, IEEE Transactions On Energy Conversion, Vol. 19, No. 3, 2004, pp: 469-476.

[3] Chang, W. F., Wu, F. Y., Chiu, C. W., Development of a web-based remote load supervision and control system, Electrical Power and Energy Systems, Vol. 28, pp: 401407, 2006.

[4] Corrado, M., Vito, L. D., Ramos, H., Saliga, J., Hardware and software platform for ADCWAN remote laboratory, Measurement, Vol.45, pp: 795-807, 2012.

[5] Hua, J., Lin, X., Xu, I., Li, J., Ouyang, M., Bluetooth wireless monitoring, diagnosis and calibration interface for control system of fuel cell bus in Olympic demonstration, Journal of Power Sources, Vol.186, pp:478-484, 2009.

[6] Huang, L. C., Chang, H. C., Chen, C. C., Kuo, C. C., A ZigBee-based monitoring and protection system for building electrical safety, Energy and Buildings, Vol. 43, pp:1418-1426, 2011.

[7] Neumann, P., Communication in industrial automationWhat is going on?, Control Engineering Practice Vol. 15, pp: 1332-1347, 2007.

[8] Huida, L., Bingchen, F., Liheng, Y., Yanan, L., RFIDBased Hazardous Waste Management Platform Establishment, Procedia Engineering, Vol.29, pp: 4-8, 2012.

[9] Ye, H. Z., Peng, J., Design of Wireless Gateway Based on ZigBee and GPRS Technology, Computational Intelligence and Software Engineering International Conference (CiSE 2009), pp:1-4, 2009.
[10] Li, N., Liu, X., Deng, Z., Su, P., Research for remote management and configuration protocol of RFID Mobile Reader, International Conference on Computer Application and System Modeling (ICCASM 2010), Vol.7-448-451, 2010.

[11] Wancheng, X., Monitoring System of Power Line Icing Based on GPRS, 2012 International Conference on Medical Physics and Biomedical Engineering, Vol. 33, pp:705 711, 2012.

[12] Gagliarducci, M., Lampasi, D.A., Podesta, L., GSM-based monitoring and control of photovoltaic power generation, Measurement, Vol. 40, pp: 314-321, 2012.

[13] Bekiroglu, E., Daldal, N., Remote control of an ultrasonic motor by using a GSM mobile phone, Sensors and Actuators A, Vol.120, pp: 536-542, 2005.

[14] Filibeli, M. C., Ozkasap, O., Civanlar, M. R., Embedded web server-based home appliance networks, Journal of Network and Computer Applications, Vol.30, pp: 499-514, 2007.

[15] Chunguo, J., Yan, W., Xin, S., Development of an OPC Server for Remote Monitoring and Control Based on GPRS Networks, The Tenth International Conference on Electronic Measurement \& Instruments (ICEMI'2011), pp:222-226, 2011.

[16] Lieping, Z., Aiqun, Z., Yunsheng, Z., On Remote Real-time Communication between MATLAB and PLC Based on OPC Technology, Proceedings of the $26_{\text {th }}$ Chinese Control Conference,pp: 545-548, 2007.

[17] Sangeetha, A. L., Naveenkumar, B., Balaji Ganesh, A., N. Bharathi, Experimental validation of PID based cascade control system through SCADA-PLC-OPC and internet architectures, Measurement, Vol.45, pp:643-649, 2012.

[18] Kong, S. C., Yeung, Y. Y., Wu, X. Q., An experience of teaching for learning by observation: Remote-controlled experiments on electrical circuits, Computers \& Education, Vol.52, pp: 702-717, 2009.

[19] Canfora, G., Daponteb, P., Rapuano, S., Remotely accessible laboratory for electronic measurement teaching, Computer Standards \& Interfaces, Vol.26, pp:489-499, 2004.

[20] Xiaorong, C., Zhan, S., Zhenhua, G., Research on Remote Data Acquisition System Based on GPRS, The Eighth International Conference on Electronic Measurement and Instruments(ICEMI'2007), Vol.2, pp:20-23, 2007.

[21] Wang, J., Zhong, S., Li, X., Wang, F., Development of 20 Liter Spherical Explosion Test Apparatus Based on LabVIEW and OPC Technique", Chinese Control and Decision Conference, pp: 4262-4266, 2010.

[22] Krejcar, O., Konarik, P., Remote Wireless Low Cost RT Process Control using Windows PLC by MATLAB/Simulink throw the REX Control System, 5th GCC Conference \& Exhibition, pp:1-5, 2009.

[23] Fang, J., Wang,F., Design of Greenhouse Remote Monitoring System based on Labview, Computer Science and Automation Engineering (CSAE), pp: 536-539, 2011.

[24] Antony, J., Mahato, B., Sharma, S., Chitranshi, G., A Web PLC using distributed web servers for data acquisition and control: Wed based PLC, Information Science and Applications (ICISA), pp: 1-4, 2011.

[25] Goel, A., Mishra, R. S., Remote Data Acquisition Using Wireless - Scada System, International Journal of Engineering (IJE), Volume (3) : Issue (1), pp: 58-65.

[26] Al-Khudairy, T. F., Al-Hashemy, B. A. R., Al-Baker M. A. 
J., Design of a VMEbus-based programmable logic controller(PLC), Microprocessors and Microsystems, Vol.21, pp:329-336, 1998.

[27] http://www.item.ntnu.no/fag/tm8100/Pensumstoff2004/GP RS_Tutorial.pdf.

[28] Alagöz, F., Mobile Networks and Data Access Strategies, Kahramanmaraş Sütçü İmam University, Institute of Science, partment of Electrical and Electronics Engineering, Master Thesis, 2005.
[29] Velagic, J., Kaknjo, J. A., Osmic, N., Dzananovic, T., Networked Based Control and Supervision of Induction Motor Using OPC Server and PLC, 53rd International Symposium ELMAR-2011, pp: 251-255, 2011.

[30] Anwar, M. R., Anwar, O., Shamim, S. F., Zahid, A. A., Human Machine Interface Using Opc (Ole For Process Control), Engineering, Sciences and Technology, Student Conference,pp:35-40,2004. 